\title{
SH 2-128, AN H II AND STAR FORMING REGION IN AN UNLIKELY PLACE
}

\author{
JOAQUIN BOHIGAS AND MAURICIO TAPIA \\ Instituto de Astronomía, UNAM, Carr. Tijuana-Ensenada Km. 10322860 Ensenada, B.C., México \\ E-mail: jbb@astrosen.unam.mx \\ (Received July 31, 2004; Accepted November 13, 2004)
}

\begin{abstract}
Near-infrared imaging photometry supplemented by optical spectroscopy and narrow-band imaging of the H II region Sh 2-128 and its environment are presented. This region contains a developed H II region and the neighboring compact H II region S $128 \mathrm{~N}$ associated with a pair of water maser sources. Midway between these, the core of a CO cloud is located. The principal ionizing source of Sh 2-128 is an O7 star close to its center. A new spectroscopic distance of $9.4 \mathrm{kpc}$ is derived, very similar to the kinematic distance to the nebula. This implies a galactocentric distance of $13.5 \mathrm{kpc}$ and $\mathrm{z}=550 \mathrm{pc}$. The region is optically thin with abundances close to those predicted by galactocentric gradients. The $J H K_{s}$ images show that S $128 \mathrm{~N}$ contains several infrared point sources and nebular emission knots with large near-infrared excesses. One of the three red $K_{s}$ knots coincides with the compact $\mathrm{H}$ II region. A few of the infrared-excess objects are close to known mid- and far-infrared emission peaks. Star counts in $J$ and $K_{s}$ show the presence of a small cluster of B-type stars, mainly associated with S $128 \mathrm{~N}$. The $J H K_{s}$ photometric properties together with the characteristics of the other objects in the vicinity suggest that Sh 2-128 and S $128 \mathrm{~N}$ constitute a single complex formed from the same molecular cloud, with ages $\sim 10^{6}$ and $<3 \times 10^{5}$ years respectively. No molecular hydrogen emission was detected at $2.12 \mu \mathrm{m}$. The origin of this remote star forming region is an open problem.
\end{abstract}

Key words : ISM:INDIVIDUAL (Sh 2-128) - ISM:H II regions - stars: formation

\section{INTRODUCTION}

Sh 2-128 (henceforth S 128) is an H II region (Sharpless 1959; Sabbadin, Strafella \& Bianchini 1986) that has been often confused as a planetary nebulae (A 77). The central star (spectral type O7 V; Chini and Wink 1984; Mampaso et al. 1984; Tylenda et al. 1991) provides almost all the photons needed for the ionization of the H II region (Haschick and Ho 1985). According Chini and Wink (1984) the distance to the star is $8.3 \mathrm{kpc}$, very similar to the kinematic distance to the nebula (Ho, Haschik \& Israel 1981). Bohigas \& Tapia (2003) rederived the distance to the star, and found it is $9.4 \pm 0.4 \mathrm{kpc}$, which implies that the galactocentric distance of $\mathrm{S} 128$ is $\simeq 13.5 \mathrm{kpc}$ and $z \simeq 550 \mathrm{pc}$, several times larger than the scale height of the molecular gas. Ho et al. (1981) found the same radio and optical morphologies and a compact H II region (S 128N) some $50^{\prime \prime}$ (2 pc) to the north. Haschick \& Ho (1985) located two $\mathrm{H}_{2} \mathrm{O}$ maser sources separated $\sim 15^{\prime \prime}$ at nearly opposite sides of the compact radio H II region. The common radial velocity of all line emitters suggests a physical relation between these objects. An elongated $29 \times 9 \mathrm{pc}$ $\mathrm{CO}$ cloud with peak emission close to $\mathrm{S} 128 \mathrm{~N}$ and a total mass of $4 \times 10^{3} \mathrm{M}_{\odot}$ is at the same kinematic distance (Ho et al. 1981; Haschick \& Ho 1985). Finally, Mampaso et al. (1984) found near-infrared emission from $\mathrm{S} 128$ and its exciting star and a second redder source

Proceedings of The 2nd Korea-Mexico Joint Workshop on Physics of the Diffuse Interstellar Medium close to $\mathrm{S} 128 \mathrm{~N}$. Both show significant excess emission at $\lambda>2.0$.

To further understand the conditions that lead to star formation at high Galactic latitudes in the outer part of the Galaxy, we obtained broad-band nearinfrared images and photometry, narrow-band optical images and optical spectroscopy of this complex. A full account of this work can be found in Bohigas \& Tapia (2003). In this contribution we present our main results.

\section{OBSERVATIONS}

\section{(a) Optical spectroscopy}

Optical spectroscopy from $3700 \AA$ to $6850 \AA$ was obtained with the $2.1 \mathrm{~m} \mathrm{f} / 7.5$ telescope of the Observatorio Astronómico Nacional at San Pedro Mártir, B.C., Mexico (OAN). The mean spectral resolution and dispersion are $\sim 3$ pixel and $2.05 \AA$ /pixel respectively (the pixel size is $24 \mu \mathrm{m}$ ). The extraction aperture is $33^{\prime \prime} \times 3.5^{\prime \prime}$ (NS and EW). Its position has been marked in Figure 1. Reddening corrections were performed with Seaton's (1979) extinction law assuming that $\mathrm{I}(\mathrm{H} \alpha) / \mathrm{I}(\mathrm{H} \beta)=2.8$.

\section{(b) Optical imaging}

Images of S 128 centered at [N II] 6584 and [O III] 5007 are presented in Fig. 1. These were secured with the $1.5 \mathrm{~m}$ telescope at the OAN and the RUCA 


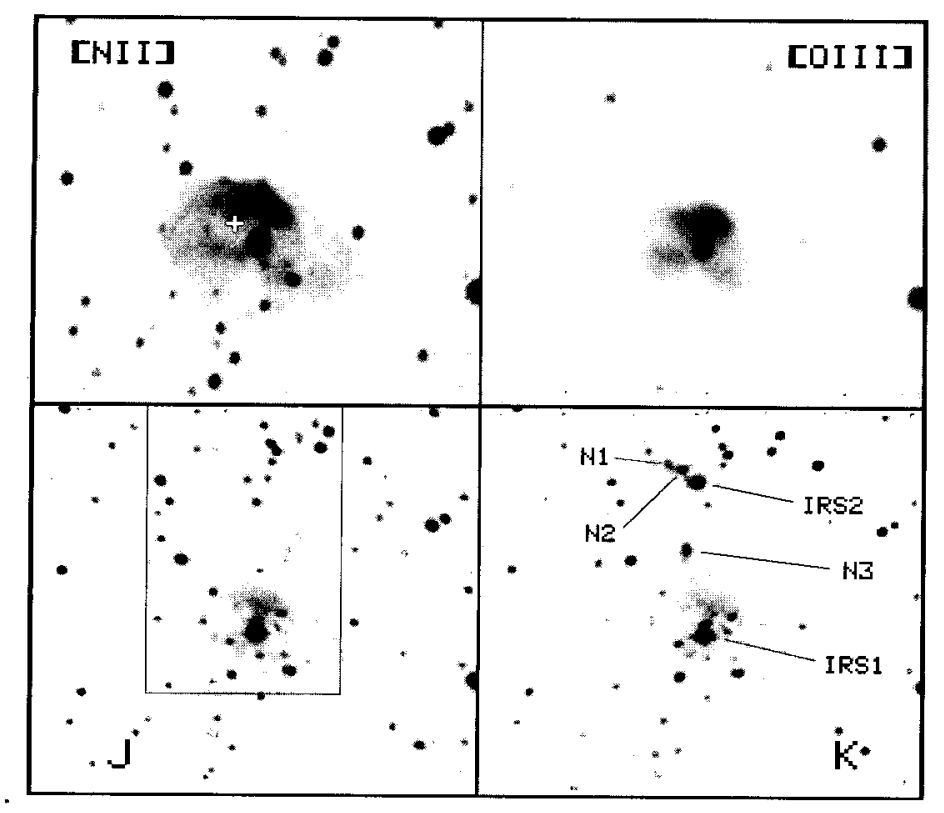

Fig. 1.- [N II] 6584 (upper left), [O III] 5007 (upper right), $J$ (lower left) and $K_{s}$ (lower right) images of S 128 . The field-of-view is $144^{\prime \prime} \times 144^{\prime \prime}$, north is up, east is to the left. The position where the optical spectrum was extracted is marked with a plus sign on the [N II] 6584 image. The region where we carried out detailed infrared photometric measurements is the rectangle in the $J$ frame. The most conspicuous near-IR sources are labelled on the $K_{s}$ image.

filter wheel (Zazueta et al. 2000). An $\mathrm{H} \alpha$ image (similar to the [N II] 6584 image) was obtained to determine the total $\mathrm{H} \alpha$ flux. Image quality (FWHM of stars) is about $1.3^{\prime \prime}$.

\section{(c) Near-infrared imaging and photometry}

Infrared observations were made with the CAMILA imaging system (Cruz-González et al. 1994) attached to the $2.1 \mathrm{~m} \mathrm{f} / 13.5$ telescope at the OAN. Images were obtained in the $J H K_{s}$ photometric bands and, in order to look for molecular hydrogen, through the $H_{2} 2$ and $c K$ narrow-band filters. Image quality (FWHM) was less than 2 pixels in all cases $\left(0.85^{\prime \prime} / \mathrm{pixel}\right)$. Series of nine overlapping frames in each filter were used to produce a $314^{\prime \prime} \times 314^{\prime \prime}$ field. The $J$ and $K_{s}$ images of the central $144^{\prime \prime} \times 144^{\prime \prime}$ are presented in Fig. 1. The region occupied by S 128 and S $128 \mathrm{~N}$ is the only site of recent star formation in the surveyed area (Haschick and Ho 1985; Hunter et al. 2000). We defined this region to be delimited by the rectangle shown in the $J$ band image in Fig. 1. The galactic field is to the total surveyed area minus this region. Compared to the field, there is an excess of $24(2.7 \sigma)$ sources in $J$ and $29(1.4 \sigma)$ in $K_{s}$ in the star forming region, statistical evidence of the presence of a small young cluster associated to the S 128 complex.

The near-infrared properties of the cluster are derived from the color-color and color-magnitude diagram (Fig. 2). About half of the sources are expected to be members of the young complex. Indeed, a large fraction of sources occupy positions in the color-color and magnitude-color diagrams that indicate infrared excess. Only one third of the sources in the star forming region are within the $\mathrm{H}$ II region $\mathrm{S} 128$, while the rest are inside the molecular cloud, though the sizes of both areas are similar. Due to higher extinction, the star density close to the peak of the CO cloud drops sharply on the $J$ image.

\section{S 128}

The faintness of [O I] $6300 \AA$ and the low $\mathrm{O}^{0} / \mathrm{O}$ ratio imply that S 128 is optically thin. The electron density and temperature in the region are $275 \pm 90 \mathrm{~cm}^{-3}$ and $10400 \pm 400 \mathrm{~K}$. Abundances are as follows: $\mathrm{He}=$ $0.089 \pm 0.001, \mathrm{O}=1.66 \pm 0.27 \times 10^{-4}, \mathrm{~N}=1.25 \pm 0.12$ $\times 10^{-5}$ and $\mathrm{S}=3.51 \pm 0.59 \times 10^{-6}$. The derived oxygen and sulfur abundances are practically identical to those reported by Rudolph et al. (1997) and very similar to those found by Vílchez \& Esteban (1996). Our nitrogen abundance is smaller than the value reported by Rudolph et al. (1997), who measure the concentration of $\mathrm{N}^{+2}$ from the far-infrared [N III] $57 \mu \mathrm{m}$ line. The nitrogen abundance reported by these authors $(2.88$ $\times 10^{-5}$ with $\left.T_{e}=10000 \mathrm{~K}\right)$ is probably closer to reality. Giveon et al. (2002) measured mid-infrared lines arising from $\mathrm{Ne}^{+}, \mathrm{Ne}^{+2}, \mathrm{Ar}^{+}$and $\mathrm{Ar}^{+2}$. The neon and argon abundances resulting from their data and formulae are $\mathrm{Ne} / \mathrm{H}=1.1 \times 10^{-4}$ and $\mathrm{Ar} / \mathrm{H}<1.5 \times 10^{-6}$. The real helium abundance in the region is larger due to the presence of some neutral helium in the $\mathrm{H}^{+}$region since the effective temperature of the exciting star (an O7 V) is lower than $\sim 40000^{\circ} \mathrm{K}$ (Stasińska \& Schaerer 


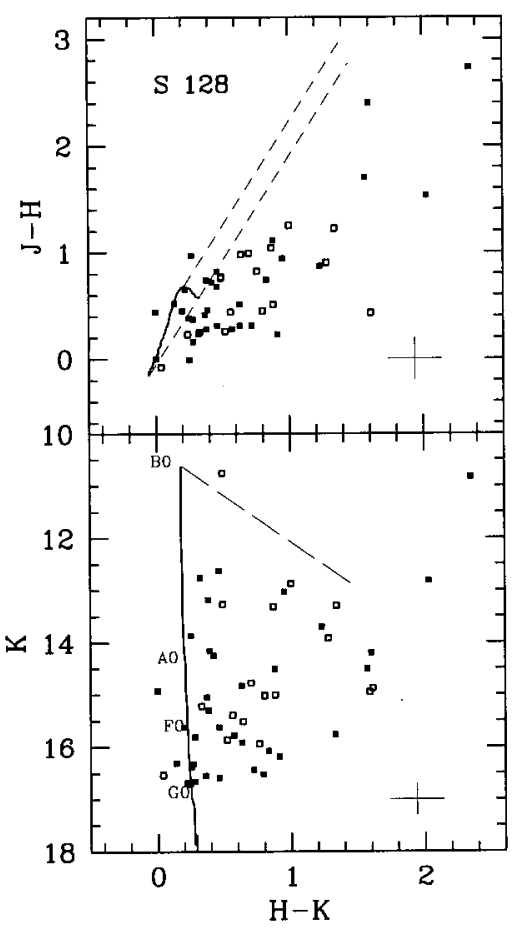

Fig. 2.- Upper panel: $J-H$ vs. $H-K_{s}$ diagram for the sources detected in these colors in the rectangular area marked in Fig. 1. The locus of main sequence stars (Koornneef 1983) is marked by the continuous line. The parallel dashed lines represent the standard reddening vectors for late and early-type stars. Lower panel: $K_{s}$ vs. $J-K_{s}$ diagram for the sources measured in $H$ and $K_{s}$ in the same area. The continuous line is the locus of the main sequence (Koornneeff 1983; Schmidt-Kaler 1983) at a distance of 9.4 kpc reddened by $A_{V}=6.1$. The long-dashed line is the standard reddening vector for a B0 star. Open symbols are for sources within the limits of the optical and radio H II region S 128, while filled symbols refer to sources located outside. The crosses illustrate the maximum photometric errors.

1997). These abundances are consistent with values determined from galactocentric gradients (Rudolph et al. 1997; Deharveng et al. 2000; Martín-Hernández et al. 2002).

From a flux calibrated (with our spectral data) $\mathrm{H} \alpha$ image we find an $\mathrm{H} \alpha$ flux of $2.8 \times 10^{-11} \mathrm{erg} \mathrm{cm}^{-2}$ $\mathrm{s}^{-1}$. With $\mathrm{C}(\mathrm{H} \beta)=2.32$ (our measured value) we find an $\mathrm{H} \alpha$ reddening corrected $\mathrm{H} \alpha$ flux of $1.6 \times 10^{-10} \mathrm{erg}$ $\mathrm{cm}^{-2} \mathrm{~s}^{-1}$. For a distance of $9.4 \mathrm{kpc}$ to $\mathrm{S} 128$ and the temperature, density and reddening corrected total $\mathrm{H} \alpha$ flux derived from our data we find that the mass of ionized hydrogen in $\mathrm{S} 128$ is close to $15 \mathrm{M}_{\odot}$. The $\mathrm{H}$ II region must receive $\simeq 1.3 \times 10^{48} \mathrm{UV}$ photons each second to ionize this mass of hydrogen at the assumed density, five times less than the rate of ionizing photons produced by the $\mathrm{O} 7 \mathrm{~V}$ central star. This is expected since the $\mathrm{H}$ II region is optically thin.

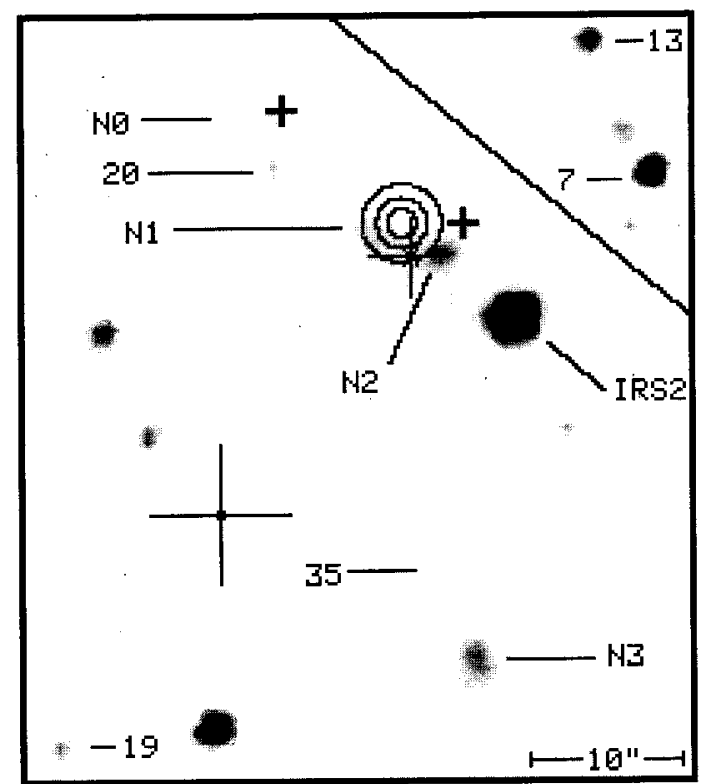

Fig. 3.- Section of the $K_{s}$-band image centered on the S $128 \mathrm{~N}$ region. North is to the top, east to the left. Concentric circles represent the $6 \mathrm{~cm}$ emission from the compact HII region (Ho et al. 1981). The small plus signs mark the positions of the two $\mathrm{H}_{2} \mathrm{O}$ masers and the square with large error bars indicates the peak emission position of the ${ }^{12} \mathrm{CO}$ cloud (Haschick and Ho 1985). The cross with error bars mark the position of the mid-infrared MSX point-source. Three near-infrared nebulous objects (N1, N2 and N3) and point sources which have $J H K_{s}$ colors that are indicative of B0 - B5 stars with significant $K_{s}$-band excess are labelled. Galactic latitude increases towards the NW and the diagonal line is at $b=3.185$

Eighteen stars were found within the limits of S 128 , six of them with spectral types B2 to B3 from their positions in the $H-K_{s}$ vs. $K_{s}$ diagram (Fig. 2), all considerably more highly reddened than the central star and with significant $K_{s}$-band excesses. The infrared properties of the stellar population probably associated with S 128 are consistent with the dynamical age of $2 \times 10^{5}$ yr of the $\mathrm{H}$ II region (Ho et al. 1981), though it may be somewhat older.

\section{S $128 \mathrm{~N}$}

The complex structure of the younger $\mathrm{S} 128 \mathrm{~N}$, which is at a projected distance $\sim 3$ pc north of $\mathrm{S} 128$; can be seen in Fig. 3, which is a $44^{\prime \prime} \times 52^{\prime \prime}$ sub-frame of our $K_{s}$-band image. The brightest source at $2.2 \mu \mathrm{m}$, Irs 2 (Mampaso et al. 1984), is dominated by a bright point-like object displaced but apparently connected to an arc-shaped series of emission knots. The brightest of these are the very red knots N1 and N2. Their relative intensities in the $H 2$ and $c K$ images are very similar, $i$. e., emission is dominated by the continuum. The compact H II region, the compact MSX source and one of the $\mathrm{H}_{2} \mathrm{O}$ masers also lie along or very close to this 
arc. The other near-infrared sources with excess $K_{s^{-}}$ band emission (sources 7, 13 and 20) are young B1-B3 reddened stars less luminous than $\mathrm{S} 128 \mathrm{~N}$.

The spectral energy distribution of the emission arising from a $30^{\prime \prime}$ area centered on $\mathrm{S} 128 \mathrm{~N}$, including the far-infrared, IRAS, MSX, $L$ and $M$ large aperture measurements and the present $J H K_{s}$ photometry of all sources in the area, can be fitted with two black-bodies with temperatures of $29 \mathrm{~K}$ and $200 \mathrm{~K}$, and a total luminosity $\geq 3.6 \times 10^{4} \mathrm{~L}_{\odot}$. The compact $\mathrm{H}$ II region is ionized by a UV flux of $5.3 \times 10^{47} \mathrm{~s}^{-1}$ (Ho et al. 1981), corresponding to a O9.5 - B0 star. This is consistent with the aforementioned total luminosity. Its $2.2 \mu \mathrm{m}$ emission probably leaks through holes in the cloud thus producing knots N1 and N2. Notice that the nebulous object $\mathrm{N} 0$ is very close to one of the water masers. The presence of water masers in the vicinity implies that the dynamical age of the compact H II region ( $\sim 10^{4}$ years; Ho et al. 1981) is not much smaller than the true age of the exciting star.

As evinced from Fig. 3 stellar formation is also occuring approximately midway between $\mathrm{S} 128$ and $\mathrm{S} 128 \mathrm{~N}$, where there are two B1-B3 type stars (sources 19 and 35 ). Close to the latter there is a very red nebulous source (N3). Its position in the color-color and magnitude-color diagrams is indicative of a highly reddened B-type star with a large infrared excess, probably an embedded star with its light scattered by dust particles in a circumstellar disk, as the morphology of N3 suggests.

\section{CONCLUSIONS}

All evidence indicates that vigorous star forming activity has been and still is occuring in S 128 and S $128 \mathrm{~N}$ for more than a million years. This massive star forming region is located in a remote region of the galaxy $13.5 \mathrm{kpc}$ away from the center and $550 \mathrm{pc}$ from the central plane - where average conditions hardly favor the formation of molecular clouds and the subsequent creation of stars (Bohigas 1988). In the standard scenario (see Hashick \& Ho 1985) the existence of this complex would be explained with a high velocity streamer falling into the plane, where it would collide and take with it a local H I cloud. The problem with this hypothesis is two-fold. The chemical composition of the region is as expected from its galactocentric distance, with no indication of a foreign contaminant, and the position and velocity of the region are consistent with the differential rotation model of the Galaxy, a situation we would hardly expect if the region originated by a streamer falling into the plane. Thus, the origin of the S 128 complex is an open question.

\section{ACKNOWLEDGEMENTS}

One of the authors $(\mathrm{JB})$ is very grateful for the warm and excellent hospitality received from all our Korean hosts, in particular Seungsoo Hong and Jong- soo Kim. Partial support from DGAPA-UNAM project IN-105400 is acknowledged.

\section{REFERENCES}

Bohigas, J. 1988, A\&A 205, 257

Bohigas, J., \& Tapia, M. 2003, AJ 126, 1861

Chini, R., \& Wink, J.E. 1984, A\&A 139, L5

Cruz-González, I. et al. 1994, Proc. of SPIE, Astronomical Instrumentation, 8, 199

Deharveng, L., Peña, M., Caplan, J., \& Costero, R. 2000, MNRAS 311, 329

Giveon, U., Sternberg, A., Lutz, D., Feuchtgruber, H., \& Pauldrach, A.W.A. 2002, ApJ 566, 880

Haschick A.D. \& Ho, P.T.P. 1985, ApJ 292, 200

Ho, P.T.P, Haschick A.D. \& Israel F.P. 1981, ApJ 243, 526

Hunter T.R., Churchwell E., Watson C., Cox P., Benford D.J. \& Roelfsma P.R. 2000, AJ 119, 2711

Koornneeff 1983, A\&A 128, 84

Mampaso, A., Gómez, P., Sánchez-Magro, C., \& Selby, M.J. 1984, MNRAS 207, 465

Martín-Hernández, N.L. et al. 2002, A\&A 381, 606

Rudolph, A.L., Simpson, J.P., Haas, M.R., Erickson, E.F., \& Fich, M. 1997, ApJ 489, 94

Sabbadin F., Strafella F. \& Bianchini A. 1986, A\&AS 65,259

Schmidt-Kaler Th. 1982, In Landolt-Börnstein, Neue Ser., p. 1, Gr VI, Vol. 2b, Stars and Star Clusters, ed. K. Schaifers \& H.H. Voight, Berlin:Springer, 10

Seaton, M.J. 1979, MNRAS 187, 73P

Sharpless, S 1959, ApJS 4, 257

Stasińska, G., \& Schaerer, D. 1997, A\&A 322, 615

Tylenda, R., Acker, A., Raytchev, B., Stenholm, B., \& Gleizes, F. 1991, A\&AS 89, 77

Vílchez, J.M., \& Esteban, C. 1996, MNRAS 280, 720

Zazueta, S. et al. 2000, RevMexAA 36141 